\title{
Ultrafast Tilting of the Dispersion of a Photonic Crystal and Adiabatic Spectral Compression of Light Pulses
}

\author{
Daryl M. Beggs, ${ }^{1,2}$ Thomas F. Krauss, ${ }^{2}$ L. Kuipers, ${ }^{1, *}$ and Tobias Kampfrath ${ }^{1,3}$ \\ ${ }^{1}$ FOM Institute AMOLF, Science Park 104, 1098 XG Amsterdam, The Netherlands \\ ${ }^{2}$ School of Physics and Astronomy, University of St. Andrews, St. Andrews, Fife, KY16 9SS, United Kingdom \\ ${ }^{3}$ Fritz Haber Institute of the Max Planck Society, Faradayweg 4-6, 14195 Berlin, Germany
}

(Received 13 July 2011; published 18 January 2012)

\begin{abstract}
We demonstrate, by theory and experiment, the ultrafast tilting of the dispersion curve of a photoniccrystal waveguide following the absorption of a femtosecond pump pulse. By shaping the pump-beam cross section with a nanometric shadow mask, different waveguide eigenmodes acquire different spatial overlap with the perturbing pump, leading to a local flattening of the dispersion by up to $11 \%$. We find that such partial mode perturbation can be used to adiabatically compress the spectrum of a light pulse traveling through the waveguide.
\end{abstract}

Physical systems can often be described by their eigenmodes, that is, states of light or matter oscillating with a well-defined frequency. For instance, the quantummechanical eigenstates of an electron spin are spin-up and spin-down [1], while the optical eigenmodes of a photonic crystal are Bloch waves characterized by their wave vector $k$ and angular frequency $\omega_{k}$ [2]. Controlling the eigenfrequencies allows for the reversible manipulation of light and matter, provided the dynamics evolve adiabatically, without exchange of energy between the eigenmodes. As an example, adiabatically changing the frequency spacing of spin states is nowadays routinely employed to cool solids [1] or to control the precession of a single spin [3]. The state-dependent change in eigenfrequencies is realized by an external magnetic or electric field which couples differently to spin-up and spin-down states.

Usually, however, optical eigenmodes (such as those of photonic crystals or microresonators) are perturbed by a homogeneous and isotropic stimulus, leading to one and the same shift of all the eigenfrequencies [4-9]. A much higher level of control could be achieved if one allows for a spatially varying perturbation that has different overlap with the light field of different modes. Such a process would result in a modified frequency spacing and density of states [10], which is particularly interesting for a photonic crystal with its continuum of modes $k$. Figure 1(a) shows that a $k$-dependent mode shift $\Delta \omega_{k}$ would lead to a local tilting of the photonic dispersion $\omega_{k}$. A light pulse being a superposition of these wave functions could then be spectrally compressed and temporally stretched, provided the dynamics were adiabatic. Moreover, as first proposed by Yanik and Fan [11], such a pulse could be also slowed down beyond the classical delay-bandwidth limitation.

In this Letter, we demonstrate that perturbing selected regions of a photonic-crystal waveguide (PCW) by means of a spatially shaped femtosecond laser pulse can locally flatten or steepen the dispersion curve. This novel procedure is akin to altering the shape of the "potential" confining the wave functions, thereby realizing a new dispersion relationship and density of states [2]. Such partial mode perturbation can be used to compress or expand the spectrum of a picosecond light pulse traveling through the waveguide. As this operation proceeds adiabatically, it is reversible and features high conversion efficiency.

Our nanophotonic approach is schematically shown in Fig. 1(b). While a light pulse travels through a PCW, a pump pulse incident from above generates an electron-hole plasma in the Si parts of the structure, thereby reducing the refractive index. The index change does not occur in the waveguide center, as a shadow mask keeps pump light away from that region. Since PCW eigenmodes have a

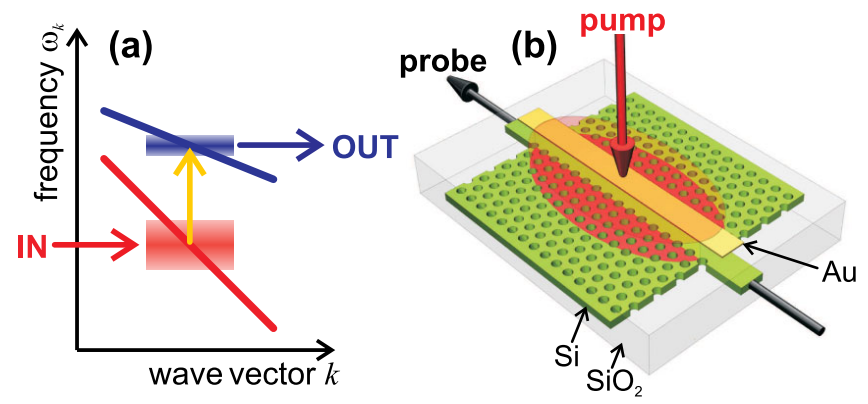

FIG. 1 (color online). (a) Schematic of adiabatic light control. An input light pulse excites eigenmodes of a photonic crystal, whose dispersion $\omega_{k}$ is then blueshifted and flattened by an external perturbation. When no light is scattered between eigenmodes, the pulse undergoes an adiabatic spectral compression. (b) Experimental realization. While a probe pulse travels through a Si-based photonic-crystal waveguide, a pump pulse generates free charge carriers in the illuminated $\mathrm{Si}$ regions. The shadow mask causes a $k$-dependent spatial overlap between the waveguide mode and pump pattern. 
strongly $k$-dependent lateral extent [Fig. 2(a)], each mode $k$ has a different spatial overlap with the pumped $\mathrm{Si}$ volume, resulting in a pronounced variation of the mode shift $\Delta \omega_{k}$ with respect to $k$. Note that the dynamics of the probe pulse inside the waveguide will proceed adiabatically because the pump pulse respects all spatial symmetries of the PCW $[8,11]$. Breaking these symmetries would result in energy transfer between different modes [12] as has been shown in a complementary experiment [13].

Theory.-We first explore our approach theoretically. To this end, we consider the frequency shift $\Delta \omega_{k}$ of mode $k$ that results when the pump pulse alters the refractive-index landscape of the photonic crystal by an amount $f(\boldsymbol{r}) \Delta n$. Here, $0 \leq f(\boldsymbol{r}) \leq 1$ reflects the normalized spatial distribution of the absorbed pump energy, which induces a change $\Delta n$ in the real part of the refractive index at positions $\boldsymbol{r}$ where $f(\boldsymbol{r})=1$. Perturbation theory [2] then predicts a relative eigenfrequency shift

$$
\frac{\Delta \omega_{k}}{\omega_{k}}=-\frac{\Delta n}{n} O_{k}
$$

which is directly proportional to the spatial overlap

$$
O_{k}=\int d^{3} \boldsymbol{r} f(\boldsymbol{r}) u_{k}(\boldsymbol{r})
$$

between the profiles of the perturbation and eigenmode. In these relations, $n=3.48$ denotes the refractive index of the unpumped $\mathrm{Si}$, and $u_{k}(\boldsymbol{r})$ is the energy density of mode $k$ normalized according to $\int d^{3} \boldsymbol{r} u_{k}(\boldsymbol{r})=1$.

In order to tilt the dispersion, $O_{k}$ needs to be $k$-dependent. More precisely, we have to realize a relative change $\Delta v_{k} / v_{k}$ in the local slope $v_{k}=\partial \omega_{k} / \partial k$ of the dispersion curve that is much larger than the perturbation $\Delta n / n$. Note that $\Delta v_{k} / v_{k}$ also quantifies the relative velocity change and relative spectral compression [Fig. 1(a)] of a light pulse encountering the dispersion change on the fly. In this respect, PCWs offer two unique benefits. First, the lateral mode extent depends strongly on $k$ as seen in Fig. 2(a) [14,15]. Second, using the slow-light modes with low group velocity $v_{k}$ means that $\Delta v_{k} / v_{k}$ can be enhanced for a given $\Delta n / n$ [8].

We calculated the eigenmodes [16] of a PCW [Fig. 1(b)], which can be pictured as a missing row of holes in a $\mathrm{Si}$ membrane (thickness $220 \mathrm{~nm}$ ), perforated with a hexagonal pattern of holes (lattice period $a=390 \mathrm{~nm}$, hole diameter $220 \mathrm{~nm}$ ). The PCW (length $L=180 a=70.2 \mu \mathrm{m}$ ) has a 600 -nm-thick $\mathrm{SiO}_{2}$ cladding on top of which a Au stripe ( $675 \mathrm{~nm}$ wide, $20 \mathrm{~nm}$ thick) serves as a shadow mask. The overlap and, thus, the interaction between the mask and the evanescent tail of light inside the waveguide are negligible. Figure 2(a) displays the unit cell of the PCW and the profile $u_{k}(\boldsymbol{r})$ of the electromagnetic energy density for several modes $k$. The respective values of $k$ can be inferred from the points labeled (i)-(xi) in the dispersion curve $\omega_{k}$ of
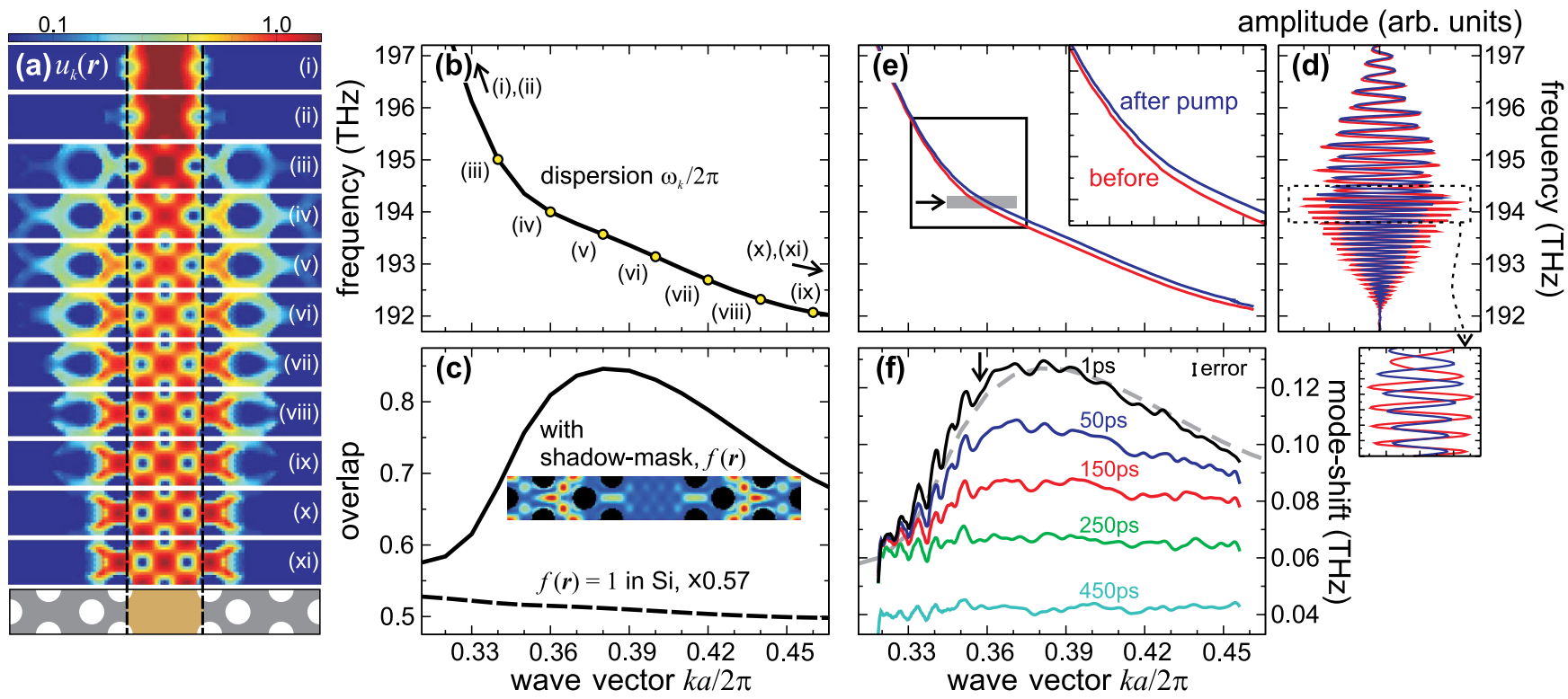

FIG. 2 (color online). Theoretical [(a)-(c)] and experimental [(d)-(f)] results for the waveguide dispersion changes. (a) Bottom panel: The shape of the unit cell of the photonic-crystal waveguide together with the shadow mask. Panels (i)-(xi): Profiles of electromagnetic energy density $u_{k}(\boldsymbol{r})$ of selected waveguide eigenmodes with $k$ indicated in (b). Note the logarithmic color scale. (b) Dispersion $\omega_{k}$ of the waveguide eigenfrequencies. (c) Solid line: Calculated overlap $O_{k}$ between the eigenmode $k$ [see (a)] and the pump pattern $f(\boldsymbol{r})$ given by the shadow mask (inset). Dashed line: $O_{k}$ for a spatially uniform change in the Si refractive index. (d) Measured real part of the Fourier-transformed output pulse $E_{o}(\omega, \tau)$ versus $\omega$ at 40 ps before (red) and 1 ps after (blue) waveguide pumping. (e) Dispersion curves of unpumped and pumped waveguides as extracted from (d). (f) Dispersion shift $\Delta \omega_{k}$ at several delay times obtained by subtraction of the measured $\omega_{k}$ [see (e)]. Dashed gray line: Theoretical curve of Fig. 2(c), repeated for comparison. Bar: Error estimated from signal variations at delays $\tau<-15$ ps. 
Fig. 2(b). With increasing $k$, the slope of $\omega_{k}$ and, thus, the group velocity decrease continuously, ending up in a flat dispersion being characteristic of slow light $\left(v_{k}<c / 50\right.$ at $k a / 2 \pi>0.45)$ [17]. As seen in Fig. 2(a), the transition from fast to slow light is accompanied by drastic changes in the spatial mode profile $[14,15]$. Whereas the light field of modes (i) and (ii) lies nearly entirely under the shadow mask, it spreads out into the surrounding lattice of holes for modes (iii)-(v) and then starts to contract again for modes (vi)-(xi).

The $k$-dependent lateral mode spread should be also reflected by the overlap $O_{k}$ of mode profile and pump pattern, as long as the mask's shadow on the Si surface is not washed out by diffraction of the pump beam. Finitedifference time-domain simulations [18] of the pump-pulse propagation indeed verify that $f(\boldsymbol{r})$ roughly follows the shape of the mask's geometric shadow [inset in Fig. 2(c)]. The resulting overlap [Eq. (2)] of $f$ with the waveguide modes is shown in Fig. 2(c) and exhibits the expected behavior: a steep rise for low wave vectors followed by a flatter decay at higher $k$. Thus, any pulse populating these modes will be spectrally compressed or expanded upon the dynamic action of the perturbation. For comparison, the dashed line shows the frequency shift for the case of a homogeneous perturbation profile $(f=1$ throughout the $\mathrm{Si})$. The $k$ dependence of $O_{k}$ nearly vanishes, because the total amount of mode energy inside the $\mathrm{Si}$ material is almost independent of $k$.

Experiment. - In order to put our partial mode perturbation scheme to the test, we fabricated a masked PCW with the same geometrical parameters used in the calculations by means of electron-beam lithography and reactive ion etching. After filling with $\mathrm{SiO}_{2}$, a Au shadow mask is placed on top of the $\mathrm{SiO}_{2}$ cladding by metal evaporation and lift-off. In order to excite the PCW, pump pulses (center wavelength $810 \mathrm{~nm}$, duration 100 fs full width at half maximum of the intensity, pulse energy $2 \mathrm{~nJ}$, and repetition rate $80 \mathrm{MHz}$ ) from a Ti:sapphire laser pass a slit and cylindrical lenses resulting in a $3-\mu \mathrm{m}$-thin line focus on the Si-membrane surface. The slit ensures exclusive and homogeneous excitation along the length of the PCW. To measure the PCW transmittance over a large bandwidth, a Fourier-limited probe pulse (1540 nm, $180 \mathrm{fs}$, and $10 \mathrm{pJ}$ ) from an optical parametric oscillator is coupled into the waveguide at a delay $\tau$ after excitation by the pump pulse. We pick up the probe at the output facet and determine the complex-valued Fourier amplitude $E_{o}(\omega, \tau)$ of its electric field by means of spectral interferometry $[19,20]$. By varying the delay between the pump and probe pulse, we obtain a two-dimensional data set $E_{o}(\omega, \tau)$.

Figure 2(d) shows the real part of the measured $E_{o}(\omega, \tau)$ at $40 \mathrm{ps}$ before PCW pumping ( $\tau=-40 \mathrm{ps}$, red line) and at $1 \mathrm{ps}$ after $(\tau=1 \mathrm{ps}$, blue line). In both cases, two regimes with slow and fast oscillations are observed.
Assuming single-mode propagation, the phase acquired by the probe pulse after propagation through the $\mathrm{PCW}$ equals $k(\omega) L$. Thus, $\operatorname{Re} E_{o}=\left|E_{o}\right| \cos (k L)$ versus $\omega$ oscillates more rapidly for slower light as the inverse group velocity $\partial k / \partial \omega$ is larger. Therefore, the fast and slow oscillations in Fig. 2(d) are signatures of slow and fast light, respectively. Solving for the phase of $E_{o}$ allows us to extract the wave vector $k$ as a function of $\omega$ (apart from an unknown offset since only phase differences can be measured). The so-obtained waveguide dispersion $\omega_{k}$ is shown in Fig. 2(e) before and after Si pumping. One clearly recognizes the regions of fast and slow light, and the shape of both curves is in very good agreement with that of the one calculated [Fig. 2(b)].

A superficial glance at Fig. 2(e) might lead to the notion that the dispersion directly after PCW pumping $(\tau=1 \mathrm{ps})$ is just a blueshifted version of the dispersion of the unpumped waveguide. However, a magnified view [inset in Fig. 2(e)] reveals departures from such rigid-shift-type behavior. This observation becomes even more apparent when we subtract one of the dispersion curves from the other. Figure 2(f) presents the resulting pump-induced mode shift $\Delta \omega_{k}$ at various delays after PCW pumping. Shortly after excitation ( $\tau=1 \mathrm{ps}$ ), the mode shift varies strongly with the wave vector, from $\Delta \omega_{k} / 2 \pi=$ $0.060 \mathrm{THz}$ (at $k a / 2 \pi=0.33$ ) to over $0.120 \mathrm{THz}(0.38)$, before dropping back to $0.095 \mathrm{THz}(0.45)$. This curve agrees excellently with the theoretical prediction [the solid line in Fig. 2(c) and the gray dashed line in Fig. 2(f)]. Comparison of the calculated overlap $O_{k}$ and the measured mode shift $\Delta \omega_{k}$ with Eq. (1) allows us to estimate the pump-induced change in the Si refractive index as $\Delta n / n \approx$ $-1.5 \times 10^{-3}$. Interestingly, at $k a / 2 \pi=0.37$ [arrows in Figs. 2(e) and 2(f)], the measured $\Delta \omega_{k}$ and, thus, $O_{k}$ have a quite steep slope, whereas the slope of $\omega_{k}$ is rather flat, resulting in a measured ratio $\left|\Delta v_{k} / v_{k}\right|$ of as much as $12 \%$. This value is more than 80 times larger than the perturbation $|\Delta n / n|$, showing that our nanophotonic approach [Fig. 1(b)] indeed causes a strongly $k$-dependent frequency shift of the photonic-crystal eigenmodes.

At later times after pump excitation, charge-carrier diffusion and recombination in the $\mathrm{Si}$ are expected to modify the refractive-index distribution $f(\boldsymbol{r}) \Delta n$ [10]. Indeed, with increasing delay $\tau$, the measured $\Delta \omega_{k}$ [Fig. 2(f)] undergoes an overall decrease and returns into a flat line $(\tau \geq$ $250 \mathrm{ps})$. This final, virtually $k$-independent mode shift agrees well with the calculated $\Delta \omega_{k}$ for a homogeneous excitation pattern [the dashed line in Fig. 2(c)]. Thus, after about $250 \mathrm{ps}$, the pump-induced charge carriers have diffused into the $\mathrm{Si}$ underneath the 675-nm-wide shadow mask, resulting in a homogeneous carrier density. These numbers allow us to estimate a mean diffusion constant of $\sim 10 \mathrm{~cm}^{2} \mathrm{~s}^{-1}$, which is in good agreement with previous measurements [21]. Whereas the observed flattening of the dispersion change arises from carrier diffusion, the 

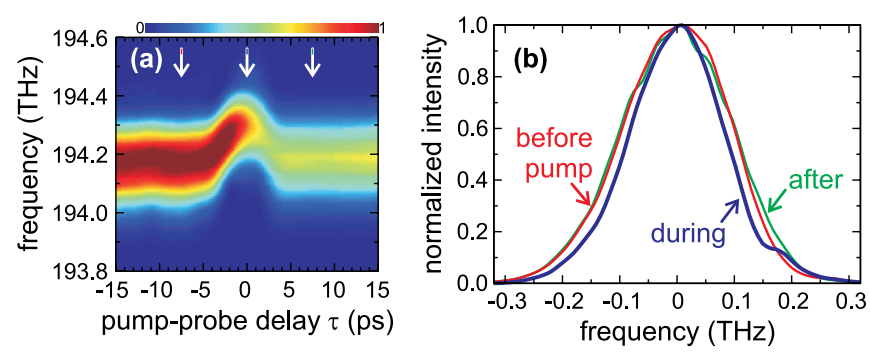

FIG. 3 (color online). Adiabatic spectral compression of a light pulse. (a) Output intensity spectra versus $\tau$ for a Fourier-limited input pulse of frequency $194.2 \mathrm{THz}$ and duration of $1.9 \mathrm{ps}$. (b) Output intensity spectra at delays indicated by arrows in (a), that is, for the mode shift occurring long before, after, or while the probe is inside the waveguide. Spectra are normalized to the same height and shifted to have center frequency $\omega=0$.

temporal decrease of the $k$-averaged $\Delta \omega_{k}$ on a time scale of about 500 ps is a consequence of carrier recombination, predominantly taking place at the surfaces of the PCW [10].

Spectral compression.-We finally illustrate how the ultrafast modifications of the PCW dispersion can be used to spectrally compress a light pulse. For this purpose, we consider a Fourier-limited Gaussian pulse with a duration of 1.9 ps and a center frequency of $194.2 \mathrm{THz}$ as PCW input [the arrows in Figs. 2(e) and 2(f)] for the following two reasons. First, the spectrum coincides with a part of the dispersion that undergoes a significant pump-induced flattening $\Delta \boldsymbol{v}_{k} / \boldsymbol{v}_{k}$ (see above). Second, this pulse allows one to confine $96 \%$ of its energy within the length of the waveguide as its duration is significantly shorter than its transit time of 5.6 ps.

We are able to determine the PCW response to the fictitious 1.9-ps input pulse from the two-dimensional data set $E_{o}(\omega, \tau)[$ Fig. 2(d)] that was measured by using the much more broadband 180 -fs probe pulse. This approach $[8,20]$ merely presumes that the waveguide response to the weak probe pulse is linear, which we have verified experimentally. The extracted output spectrum is shown in Fig. 3(a) as a function of the delay between the pump and probe pulse. At delays $\tau<-5$ ps and $\tau>5 \mathrm{ps,}$ no dynamics occur as the probe pulse encounters a fully unexcited or excited waveguide, respectively. The energy of the output pulse is smaller in the excited case, because free charge carriers lead to additional light absorption. At $\tau=0$, the probe pulse is fully contained in the PCW when the pump-induced shift $\Delta \omega_{k}$ of the dispersion curve occurs. As the light populating these modes is frequencyshifted as well [Fig. 1(a)], we find a clear blueshift of the PCW output around $\tau=0$ [Fig. 3(a)]. The center frequency increases by $0.12 \mathrm{THz}$, in good agreement with the magnitude of the measured $\Delta \omega_{k}$ [Fig. 2(f)].

In order to evaluate further spectral modifications of the blueshifted pulse, Fig. 3(b) displays the extracted output spectra before and after waveguide excitation as well as at $\tau=0$ [see the arrows in Fig. 3(a)]. For better comparison, the spectra are normalized to the same height, and their center frequencies are shifted to frequency $\omega=0$. Note that the spectrum of the blueshifted pulse is noticeably narrower than the spectra obtained before and after PCW pumping. The relative spectral decrease of the full width at half maximum amounts to about $11 \%$, which agrees well with the relative change $\Delta v_{k} / v_{k}$ of $12 \%$ of the slope of the dispersion curve [Figs. 2(e) and 2(f)]. Therefore, our findings are consistent with the adiabatic spectral compression of light as anticipated in Fig. 1(a). The conversion efficiency is better than $60 \%$ and limited only by free-carrier absorption. We note that adiabatic spectral expansion can also be obtained when pulses in the slow-light region at 193.3 THz [Fig. 2(e)] are used (data not shown).

In conclusion, we have demonstrated that partial mode perturbation can be used for all-optical adiabatic pulse-bandwidth compression. This process is significant because it overcomes the fundamental bandwidth-delay constraint in optics [11]. Ultimately, such a process can generate arbitrarily small group velocities for any light pulse with a given bandwidth, without the need for intrinsic material resonances. The unavoidable signal loss due to free-carrier absorption could be prevented by using tuning mechanisms like the instantaneous optical Kerr effect. Besides the dynamical slow-down of light, spectral compression could also find application as a magnifying time lens [22-24]: The uniform shrinking of the frequency axis by a factor $1+\Delta v_{k} / v_{k}$ yields an expansion of the pulse by the inverse factor in the time domain. The magnitude of the spectral compression could be enhanced further by optimizing the profiles of the perturbing pump or by modifying the photonic crystal itself. One could repeat the spectral compression in cascaded photonic crystals: The smaller the bandwidth of the pulse becomes, the smaller the slope of the dispersion of the next waveguide stage can be chosen, leading to a larger relative compression $\Delta v_{k} / v_{k}$.

We acknowledge funding through the EU FP6-FET "SPLASH" project. This work is also part of the research program of FOM, which is financially supported by the NWO.

*kuipers@amolf.nl

[1] C. Kittel, Introduction to Solid State Physics (Wiley, New York, 2005).

[2] J. D. Joannopoulos, Photonic Crystals: Molding the Flow of Light (Princeton University, Princeton, NJ, 2008).

[3] J. Berezovsky, M.H. Mikkelsen, N. G. Stoltz, L. A. Coldren, and D. D. Awschalom, Science 320, 349 (2008).

[4] D. A. Mazurenko et al., Phys. Rev. Lett. 91, 213903 (2003).

[5] Y. A. Vlasov, M. O’Boyle, H. F. Hamann, and S. J. McNab, Nature (London) 438, 65 (2005).

[6] Q. Lin, T. J. Johnson, C. P. Michael, and O. Painter, Opt. Express 16, 14801 (2008). 
[7] T. Tanabe, M. Notomi, H. Taniyama, and E. Kuramochi, Phys. Rev. Lett. 102, 043907 (2009).

[8] T. Kampfrath et al., Phys. Rev. A 81, 043837 (2010).

[9] J. Upham, Y. Tanaka, T. Asano, and S. Noda, Appl. Phys. Express 3, 062001 (2010).

[10] H. W. Tan, H. M. van Driel, S. L. Schweizer, and R. B. Wehrspohn, Phys. Rev. B 72, 165115 (2005).

[11] M. F. Yanik and S. Fan, Phys. Rev. Lett. 92, 083901 (2004).

[12] B. A. Daniel, D. N. Maywar, and G. P. Agrawal, J. Opt. Soc. Am. B 28, 2207 (2011).

[13] P. Dong, S. F. Preble, J. T. Robinson, S. Manipatruni, and M. Lipson, Phys. Rev. Lett. 100, 033904 (2008).

[14] A. Petrov, M. Krause, and M. Eich, Opt. Express 17, 8676 (2009).

[15] L. O'Faolain et al., Opt. Express 18, 27627 (2010).
[16] We used the freely available Massachusetts Institute of Technology PHOTONIC BANDS package; see S. G. Johnson and J. D. Joannopoulos, Opt. Express 8, 173 (2001).

[17] T. Baba, Nature Photon. 2, 465 (2008).

[18] We used the commercially available software FULLWAVE by Rsoft.

[19] L. Lepetit, G. Chériaux, and M. Joffre, J. Opt. Soc. Am. B 12, 2467 (1995).

[20] T. Kampfrath, D. M. Beggs, T. F. Krauss, and L. Kuipers, Opt. Lett. 34, 3418 (2009).

[21] C.-M. Li, T. Sjodin, and H.-L. Dai, Phys. Rev. B 56, 15252 (1997).

[22] B. H. Kolner and M. Nazarathy, Opt. Lett. 14, 630 (1989).

[23] F. Biancalana, A. Amann, A. V. Uskov, and E. P. O'Reilly, Phys. Rev. E 75, 046607 (2007).

[24] M. A. Foster et al., Nature (London) 456, 81 (2008). 